\title{
Partial Preservation of the Inferior Turbinate in Endoscopic Medial Maxillectomy: A Computational Fluid Dynamics Study
}

American Journal of Rhinology \& Allergy

\author{
Alberto M. Saibene, MD, MA ${ }^{1,2}\left(\mathbb{D}\right.$, Giovanni Felisati, $M D^{1,2}$, \\ Carlotta Pipolo, MD ${ }^{1,2}$, Antonio Mario Bulfamante, $M D^{1,2}$ (D, \\ Maurizio Quadrio, $\mathrm{PhD}^{3}$, and Vanessa Covello, $\mathrm{PhD}^{2,3}$
}

\begin{abstract}
Background: Endoscopic medial maxillectomy (EMM) is a workhorse for multiple sinonasal conditions. To reduce its burden on the sinonasal physiology, several modified EMM (M-EMM) have been proposed. Objective: In order to provide a theoretical basis for EMM and its modifications, this study introduces a computational fluid dynamics (CFD) model, based on a time-resolved direct numerical simulation, describing EMM and assessing the role of the M-EMM in preserving the overall fluid dynamics of the sinonasal cavities.

Methods: A normal sinonasal CT scan was converted into a geometrical model and used as a reference; 2 anatomies were then created by virtual surgery, mimicking EMM and M-EMM, with the latter sparing the anterior portion of inferior turbinate and medial maxillary sinus wall. The airflow was simulated in the models via the OpenFOAM CFD software and compared in terms of flow rate, mean and fluctuating velocity, vorticity, and turbulent structures.

Results: The analysis shows that EMM induces a massive flow rate increase in the operated side, which becomes less obvious in the M-EMM model. In contrast to M-EMM, EMM induces higher velocity fields that reach the maxillary sinus. Velocity and vorticity fluctuations are negligible in the baseline model, but become increasingly evident and widespread in the M-EMM and EMM models.

Conclusions: A significant disruption of the nasal fluid dynamics is observed in EMM, while M-EMM minimizes variations and reduces interference with nasal air conditioning. Our analysis provides insights into the pathophysiology of radical sinus surgery and provides a theoretical basis for the ability of M-EMM to reduce the temporary surgery-related changes on both healthy and operated sides.
\end{abstract}

\section{Keywords}

endoscopy, paranasal sinuses, nasal airflow dynamics, computer modeling for nasal airflow, maxillectomy, nasal airflow, endoscopic sinus surgery, turbulence, wall shear stress, turbinates, maxillary sinus

\section{Introduction}

Since its introduction, ${ }^{1}$ endoscopic medial maxillectomy (EMM) has become a staple procedure in the management of maxillary sinus (MS) neoplasms. ${ }^{2}$ Furthered by technical refinements, ${ }^{3}$ EMM is also employed to address selected inflammatory conditions.

EMM shows solid surgical results, ${ }^{4,5}$ but it has known minor issues (crusting, lacrimal pathway obstruction, and malar region hypoesthesia). ${ }^{6}$ In order to address them, several types of modified EMM (M-EMM) have

\footnotetext{
'Unit of Otolaryngology, ASST Santi Paolo e Carlo, Milan, Italy

${ }^{2}$ Department of Health Sciences, Università degli Studi di Milano, Milan, Italy

${ }^{3}$ Department of Aerospace Sciences and Technologies, Politecnico di Milano, Milan, Italy

Corresponding Author:

Alberto M. Saibene, Otolaryngology Department, San Paolo Hospital, Via Antonio di Rudini, 8, 20142 Milan, Italy.

Email: alberto.saibene@gmail.com
} 
been proposed, ${ }^{7-10}$ aimed at sparing the lacrimal pathway and the inferior turbinate (IT).

IT preservation is commonly thought to preserve its role in temperature adjustment and nasal airflow control. ${ }^{11,12}$ Conversely, whole turbinate resections lead to persistent crusting and reduction of inhaled air conditioning. ${ }^{7}$ Such observations are supported by anecdotal reports and subjective surgical outcomes analysis, ${ }^{7,13}$ but no study thoroughly addressed the role of IT resection and partial preservation in EMM from a fluid dynamical standpoint.

Few literature studies provided numerical models of EMM built via computational fluid dynamics (CFD) ${ }^{14,15}$ In particular, Lindemann et al. ${ }^{14}$ hint at the presence of large vortical structures in the MS after EMM. Such CFD studies are limited by their computational design, relying on rather simple mathematical models, unable to simulate the fluctuating quantities. While the IT role has been studied with different in vitro and computational models, ${ }^{16,17}$ suggesting the appearance of chaotic and/or vortical flow patterns in the nasal cavity after aggressive turbinate resections, no studies mirrored these changes in EMM patients.

This study aims to present an advanced CFD model, based on a time-resolved direct numerical simulation (DNS) approach, able to fully describe EMM and to assess the role of partial IT preservation in the overall fluid dynamics of the sinonasal cavities. A solid theoretical foundation for EMM modifications will thus be provided.

\section{Materials and Methods}

At odds with the majority of available CFD studies, our study is based on a DNS. DNS solves numerically the equations governing the air motion without resorting to a turbulence model. The numerical solution is thus truly three dimensional and unsteady, and the mean fields and their statistics are computed a posteriori by a process of time-averaging akin to a real experimental measurement.
The airflow in the reconstructed nasal cavities was simulated via the open-source CFD software OpenFOAM. ${ }^{18}$ The numerical approach, already demonstrated in previous work, ${ }^{19,20}$ included a Large Eddy Simulation turbulence model; however, its contribution was negligible here thanks to the fine mesh employed, capable to capture all the spatial-temporal motion scales.

After approval by the internal Institutional Review Board of the San Paolo Hospital, University of Milan, the scan of a 67-year-old man presenting a normal sinonasal anatomy was selected. The CT scan contained 348 DICOM images, with spatial resolution of $0.5 \mathrm{~mm} \times 0.5 \mathrm{~mm}$ in the sagittal-coronal directions and a $0.6-\mathrm{mm}$ axial gap between consecutive slices. CT images were converted into an accurate geometrical model via the open-source software 3D-Slicer, ${ }^{21}$ choosing a proper radiodensity threshold. ${ }^{22}$ A 3D computational domain was then built, according to the procedure illustrated in previously published papers. ${ }^{23}$

The anatomy reconstruction was used as the baseline pre-op reference and formed the basis for 2 additional virtual surgery anatomies (see Results for details), providing a model of standard EMM and M-EMM. Each of the 3 cases was then discretized onto a 50-million cell volume mesh, corresponding to an average spatial resolution of the order of $200 \mu \mathrm{m}$.

The 3 cases were simulated under the same conditions employing the DNS of the Navier-Stokes equations to assess the postoperative outcomes: a steady inspiration, lasting 0.6 seconds after statistical equilibrium, driven by a pressure difference of $20 \mathrm{~Pa}$ between the external ambient and the laryngeal region. The simulation employed no-slip and no-penetration boundary conditions at the wall and a zero-gradient velocity boundary condition at the outlet. With a time step size of $1 \times 10^{-5}$, each simulation required $6 \times 10^{4}$ steps to complete.

Using the 3DSlicer software, the original 3D model was reprocessed to obtain 2 virtual surgery models (see Figure 1). The 2 models represent an EMM and an M-EMM performed on the patient's left side.

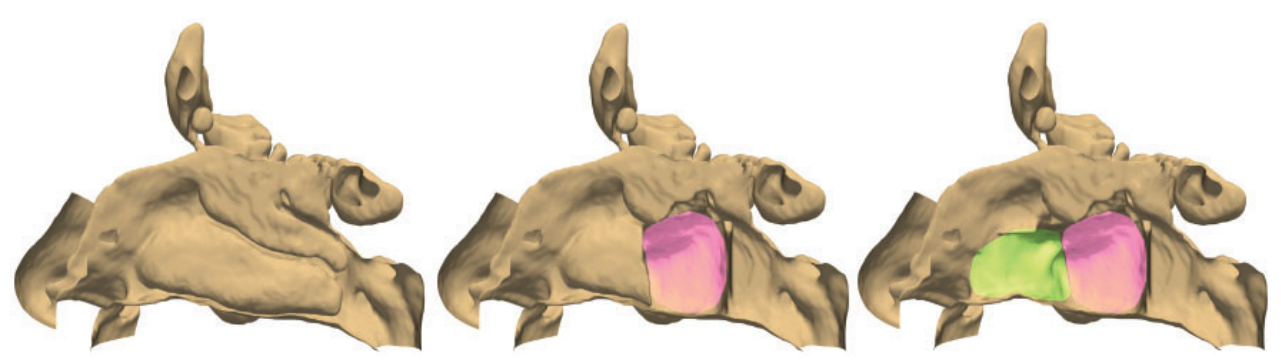

Figure I. The 3 anatomies considered in the present study: the baseline model (left), the anatomy after virtual EMM (right), and the conservative virtual M-EMM (center) where the anterior part of inferior turbinate and maxillary sinus were spared. Colors indicate the affected anatomical regions: pink highlights the lateral nasal wall demolition of M-EMM, while green indicates the further demolition required for EMM. EMM, endoscopic medial maxillectomy; M-EMM, modified endoscopic medial maxillectomy. 
In the EMM model, mimicking common approaches for sinonasal neoplasms, we removed the whole IT, the MS medial wall, the uncinate process, and the middle turbinate, sparing its superior and lateral insertions; the ethmoid bulla was opened.

In the M-EMM model, the anterior portion of the IT and the anterior portion of the medial MS wall up to the nasolacrimal duct were spared, as proposed in various

Table I. Values for the Volumetric Flow Rate (Q) for the Baseline, M-EMM, and EMM Models.

\begin{tabular}{lccr}
\hline & Baseline & M-EMM & \multicolumn{1}{c}{ EMM } \\
\hline Q RIGHT (I/min) & 9.223 & $8.760(-5.0 \%)$ & $6.983(-24.3 \%)$ \\
Q LEFT (I/min) & 6.738 & $8.290(+23.0 \%)$ & $11.612(+72.3 \%)$ \\
Q TOT $(1 / \mathrm{min})$ & 15.961 & $17.048(+6.8 \%)$ & $18.595(+16.5 \%)$ \\
\hline
\end{tabular}

Abbreviations: EMM, endoscopic medial maxillectomy; M-EMM, modified endoscopic medial maxillectomy.

The table reports the quantitative flow rate of the nonoperated (right) side, for the operated (left) side, and the overall quantitative flow rate, for the assigned pressure drop. The flow rate increase in the operated side is less obvious in the M-EMM model. models. ${ }^{7}$ All the other modifications were replicated from the EMM model.

\section{Results}

The comparative CFD analysis of the 3 models reveals evident changes in the airflow patterns after EMM, while M-EMM presents general features more closely resembling the baseline.

\section{Volumetric Flow Rate and Velocity}

The first and foremost difference is how EMM induces a higher increase in the flow rate of the operated (left) side (see Table 1) than M-EMM model.

Second, as shown in Figures 2 and 3, in the baseline model, the highest velocity region is located in the middle meatus. EMM induces a substantial alteration of the mean velocity field in the operated side, showing higher velocity in the MS lateral and cranial portion. Furthermore, EMM reduces the maximum velocity
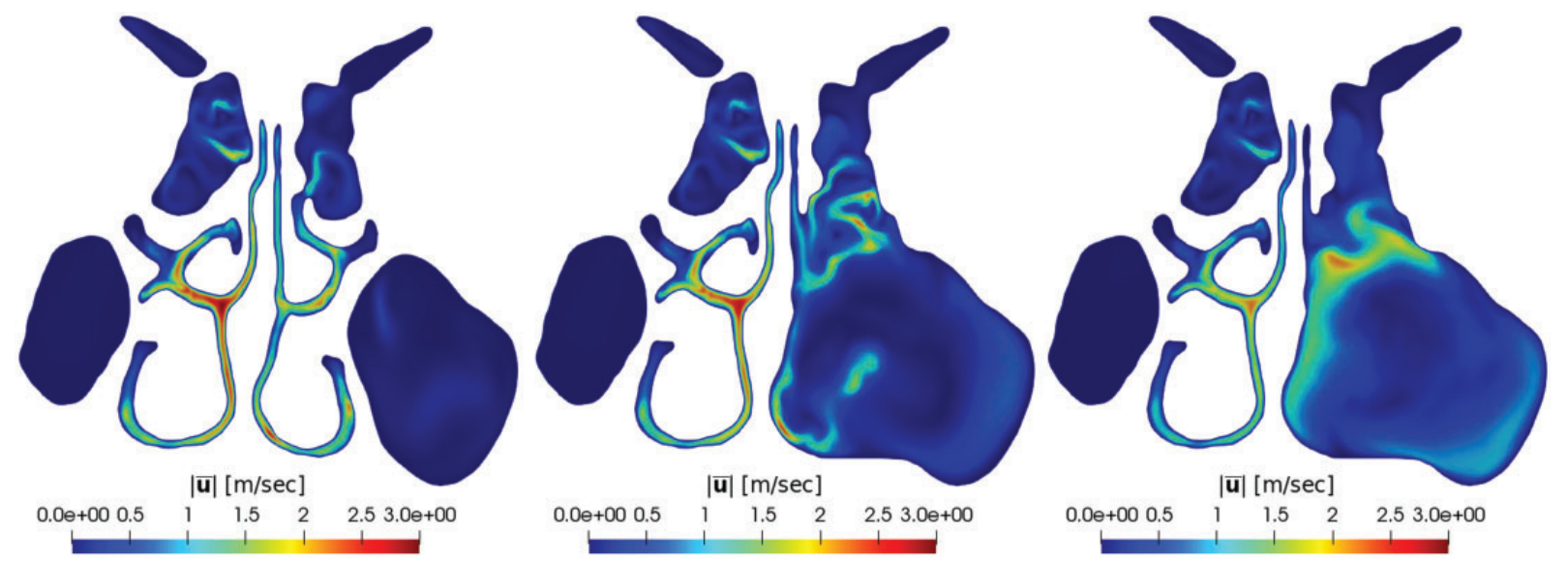

Figure 2. Magnitude of the mean velocity field in a coronal plane passing through the volume interested by surgery. From left to right: baseline, M-EMM, and EMM.
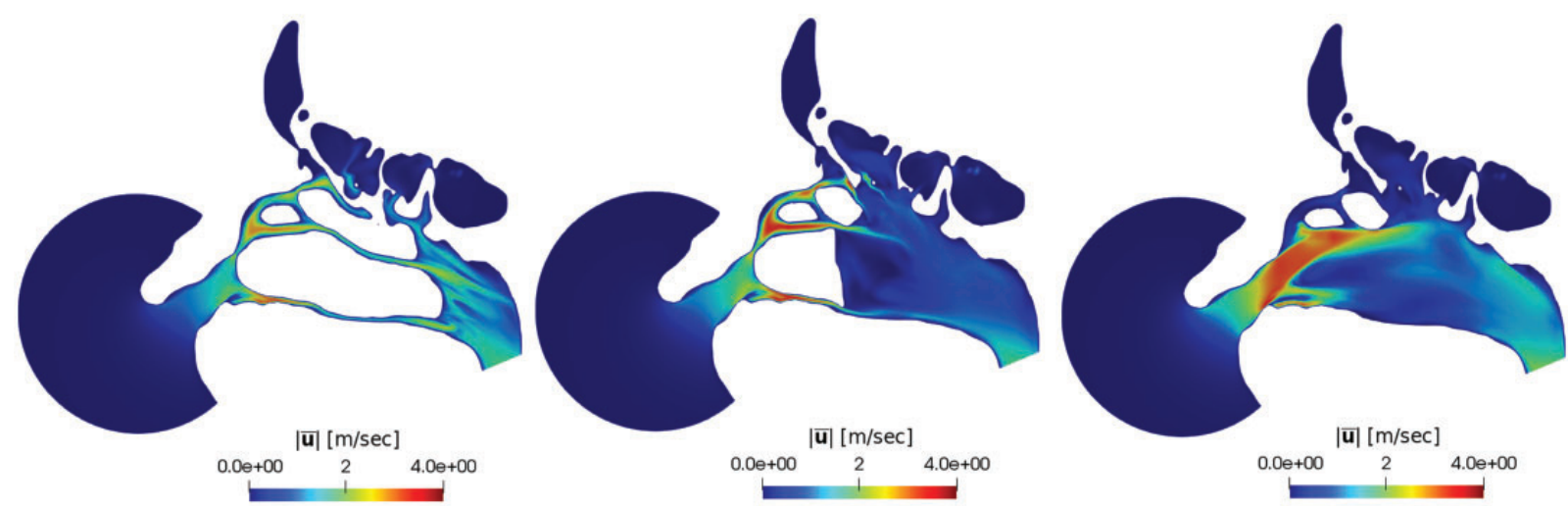

Figure 3. Magnitude of the mean velocity field in a sagittal plane. From left to right: baseline, M-EMM, and EMM. 
and overall flow rate in the contralateral side. Conversely, in M-EMM, the mean velocity field does not present increased velocity areas and the contralateral side remains essentially unchanged. Last, the larger the turbinate resection, the more streamlines penetrate the MS, as shown in Figure 4.

\section{Fluctuations}

Our time-resolved simulation provides information on flow unsteadiness, quantified here by the root-meansquare (RMS) values of the fluctuations of the velocity magnitude. As shown in Figures 5 and 6, in the baseline model, the RMS value in the inferior and middle meatus

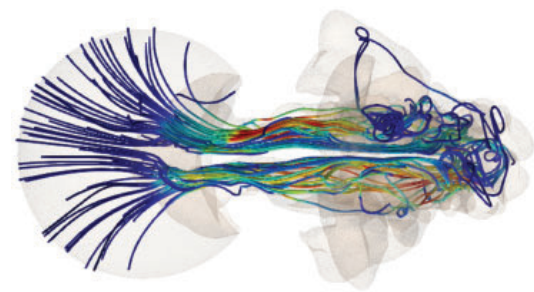

|ü $[\mathrm{m} / \mathrm{sec}]$

$0.0 e+00 \quad 1.5 \quad 3.0 e+00$

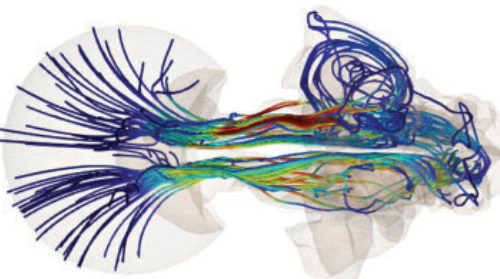

$|\mathbf{u}|[\mathrm{m} / \mathrm{sec}]$

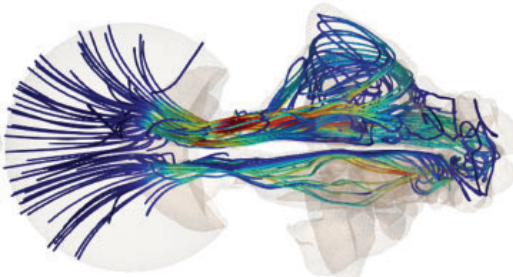

|ü $[\mathrm{m} / \mathrm{sec}]$

Figure 4. Three-dimensional view of the streamlines in the baseline (left), M-EMM (center), and EMM (right) cases. Streamlines departing from the outer ambient are observed to progressively enter the right maxillary sinus as the turbinate resection becomes more substantial. Streamlines are colored with the local magnitude of the velocity vector.
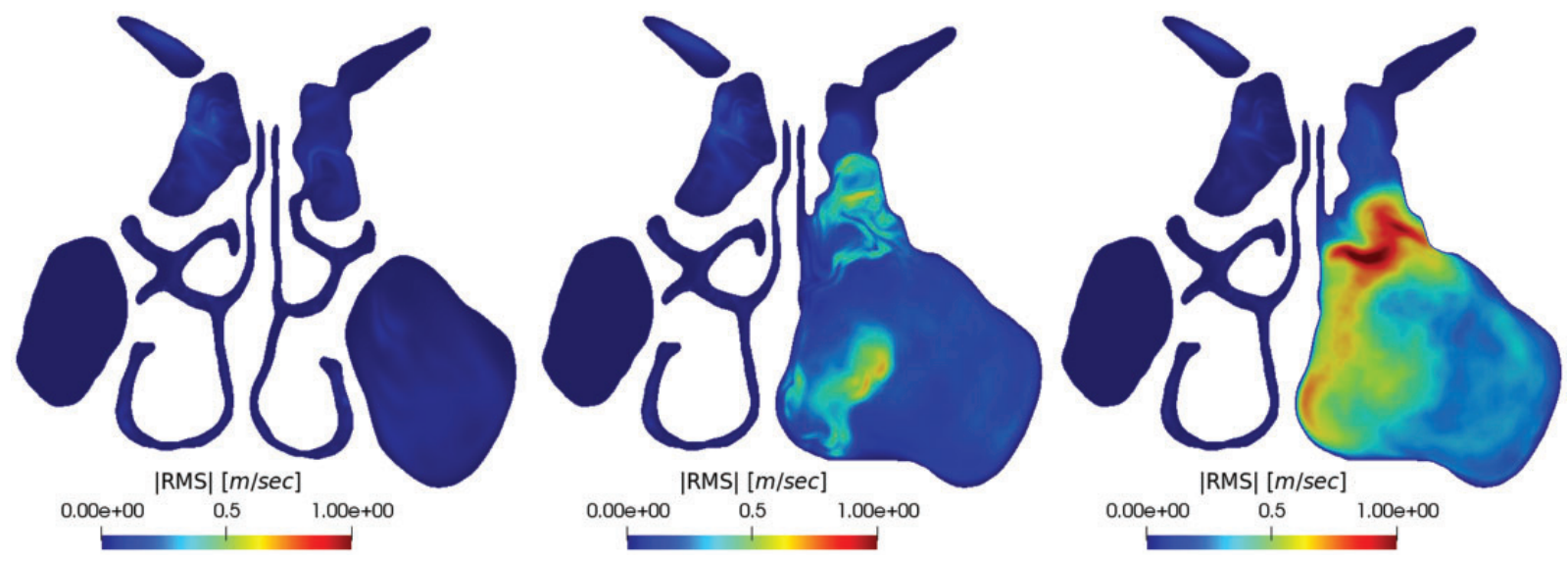

Figure 5. Root-mean-square value of the fluctuations of the velocity magnitude in a coronal plane. From left to right: baseline, M-EMM, and EMM. RMS, root-mean-square.
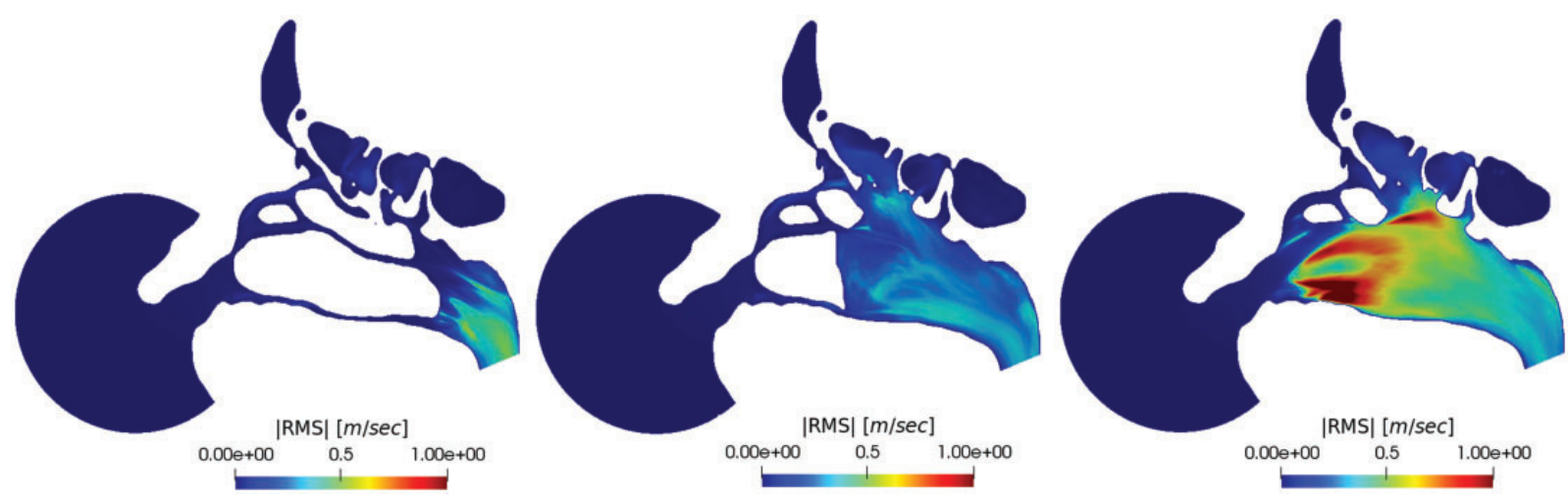

Figure 6. Root-mean-square (RMS) value of the fluctuations of the velocity magnitude in a sagittal plane. From left to right: baseline, M-EMM, and EMM. RMS, root-mean-square. 
is negligible, suggesting a nearly steady flow. In the M-EMM and EMM models, however, the velocity field shows significant fluctuations. The sites where the largest fluctuations are observed vary from M-EMM (where they are strongest just behind the IT stump) to EMM (where fluctuations are strongest in the former ethmoid/middle turbinate region). The maximum RMS value is larger in the EMM model than in the M-EMM model.

The magnitude of the vorticity vector is shown in Figure 7. It grows progressively from the baseline to the EMM model. Vorticity is observed to progressively reach the lateral MS wall in the EMM model. M-EMM contains vorticity toward the midline, reducing the MS involvement.

Table 2 shows the volumetric average of the turbulent kinetic energy $(\mathrm{K})$. Turbulence intensity increases by orders of magnitudes in the operated side and, less remarkably, in the $\mathrm{M}-\mathrm{EMM}$ model. Interestingly, the values show a definite, progressive increase through M-EMM to EMM also in the nonoperated side.

Figure 8 shows isosurfaces of the second eigenvalue $\lambda 2$ of the velocity gradient tensor. This scalar quantity is used as a proxy to visualize turbulent vortical structures; ${ }^{24}$ its spatial distribution does not provide indication of coherent large-scale vortices and vividly illustrates how the flow is severely modified by EMM (and, to a much lesser extent, by M-EMM), where the large number of vortical structures correlates to the increased flow unsteadiness observed in Figures 5 and 6.

\section{Discussion}

The role of turbinates and lateral nasal wall in air conditioning has been studied and demonstrated with in silico and in vivo studies. ${ }^{16,25,26}$ Similarly, the relationship between a failure in nasal conditioning and nasal crusting, especially after extensive surgery, has been thoroughly explored and demonstrated. ${ }^{27,28}$ However, these speculations have been only marginally extended to the EMM.

The disruption of normal nasal physiology in EMM is one of the main reasons why the more conservative M-EMM has been proposed. ${ }^{3,7}$ However, to the authors' knowledge, only 2 CFD studies have explored the effects of EMM on the nasal function, ${ }^{14,15}$ providing insight into the change of flow patterns after EMM. Both are based on a single-patient analysis. Unfortunately, Qian et al. ${ }^{15}$ used a simple mathematical approach unable to provide information on the unsteady component of the flow, whereas in Lindemann the flow was driven by an
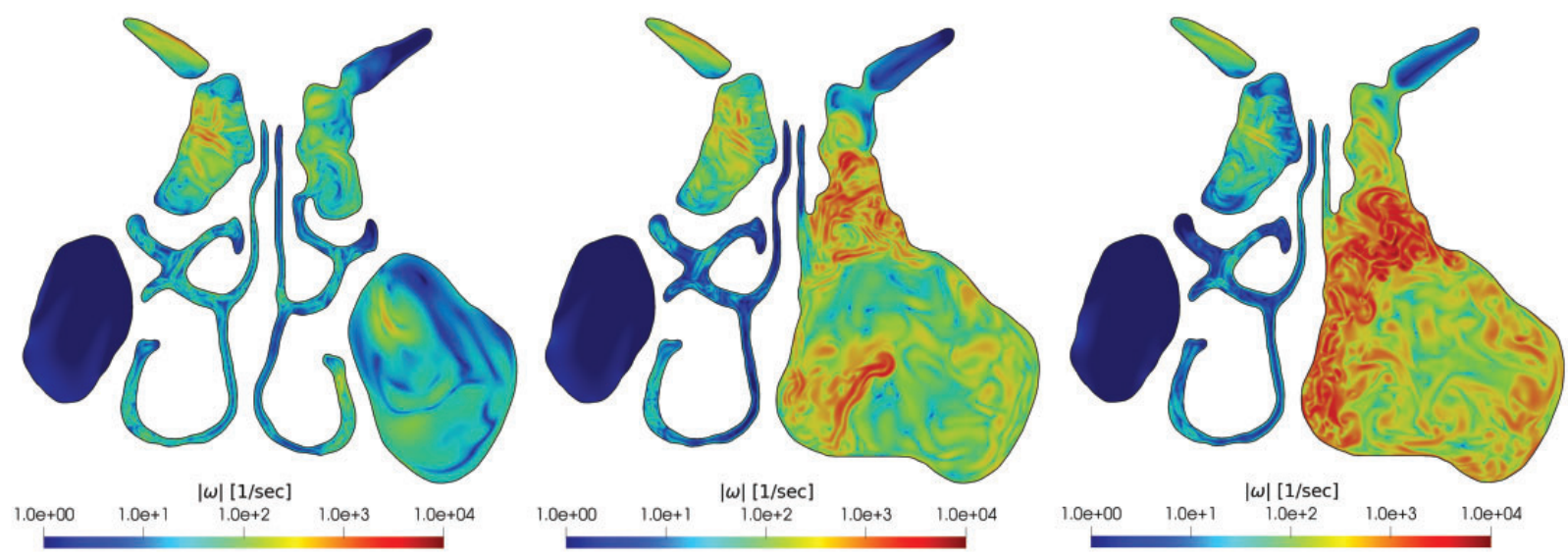

Figure 7. Magnitude of the instantaneous fluctuating vorticity vector in a coronal plane passing through the volume interested by surgery. Note the logarithmic color scale. From left to right: baseline, M-EMM, and EMM.

Table 2. Values for Turbulent Kinetic Energy Volume Integral.

\begin{tabular}{llll}
\hline & Baseline & M-EMM & EMM \\
\hline K volume average right $\left(\mathrm{m}^{2} / \mathrm{s}^{2}\right)$ & 0.000194 & $0.000301(1.5 \times)$ & $0.000845(3.5 \times)$ \\
$\mathrm{K}$ volume average left $\left(\mathrm{m}^{2} / \mathrm{s}^{2}\right)$ & 0.000165 & $0.006093(35 \times)$ & $0.028219(170 \times)$ \\
$\mathrm{K}$ volume average TOT $\left(\mathrm{m}^{2} / \mathrm{s}^{2}\right)$ & 0.000359 & $0.006394(16.8 \times)$ & $0.029064(79.9 \times)$ \\
\hline
\end{tabular}

Abbreviations: EMM, endoscopic medial maxillectomy; M-EMM, modified endoscopic medial maxillectomy.

The table reports the turbulent kinetic energy $(K)$ volume average for the nonoperated (right) side and the operated (left) side. The $\mathrm{K}$ volume average quantifies the increase of turbulence in the operated side; although with EMM the increase is by 170 times, with M-EMM the increase is only 35 times. The values show a definite, progressive increase through M-EMM to EMM also in the nonoperated side. 


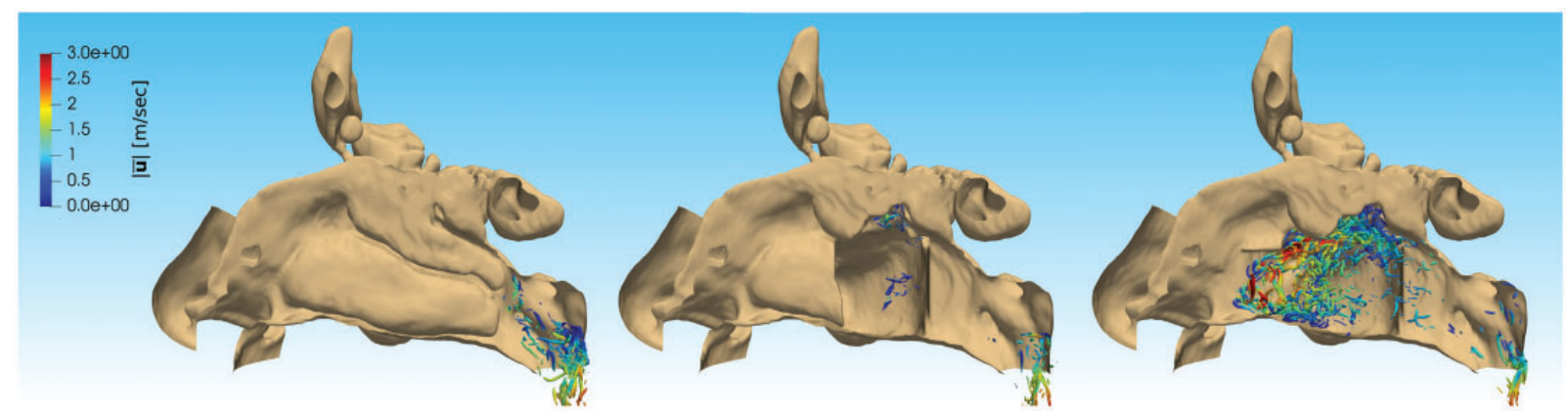

Figure 8. Isosurface of $\lambda 2=-650000 / s^{2}$ in an instantaneous velocity field, showing vortical structures, colored by the local value of the velocity vector. From left to right: baseline, M-EMM, and EMM.

extremely low pressure difference of only $1.3 \mathrm{~Pa},{ }^{14,15}$ times lower than the present study, implying a breathing intensity well below that at rest. In our work, a DNSbased and time-resolved simulation is employed to assess unsteady phenomena at a physiological breathing rate.

Even if an increase of the flow in the MS has been already thoroughly demonstrated after standard antrostomy procedures, ${ }^{29}$ our analysis finds EMM to profoundly modify how the flow rate is partitioned between the 2 nasal airways. A certain amount of unbalance is physiological ${ }^{30}$ : Indeed in our baseline case, $42 \%$ of the flow rate pertains to the right passageway and $58 \%$ to the left. Within the specific constraint of a comparison carried out for the same global pressure drop, EMM determines a massive $(+72 \%)$ relative increase of the flow rate on the operated side, with significant reduction induced in the contralateral side too. Overall, the flow rate for the same pressure drop increases by $16.5 \%$. On the other hand, M-EMM increases flow rate in the operated side much less dramatically, with minimal contralateral changes, and the end result is a nearly equilateral flow rate distribution $(49 \%$ on the right side, $51 \%$ on the left) with only $6.8 \%$ increase in the overall flow rate.

Some studies indicated nasal cavity volume as the main predictive factor for air conditioning function, together with temperature distribution along the nasal surface. ${ }^{31}$ Even in this respect, M-EMM scores better than EMM, although the significance of comparisons based on a constant pressure drop remains to be assessed. On the basis of this observation, it can be surmised that one of the main reasons of the increased crusting, bleeding, and mucosal drying could be a direct effect of the airflow unbalance, influencing both the healthy and operated sides. This hypothesis might as well explain why temporary postoperative changes affect also the unmodified contralateral side.

Our study finds that the topology of the flow field remains generally unchanged, with the sole difference that more streamlines reach the MS as the IT resection is increased. These findings are partially in contrast with data reported by Lindemann at much lower velocities. ${ }^{14}$ Indeed, we have found that air still flows freely toward the nasopharynx in both the unaffected and operated sides, both in the EMM and M-EMM models. Lindemann and colleagues showed instead (albeit at low breathing intensity) large vortical structures disrupting the normal flow in the nasal cavities; such structures have some similarities with the vortices induced by radical turbinectomy in the solid model proposed by PérezMota et al. ${ }^{17}$ According to their findings, such vortices prevented the correct flow toward the nasopharynx, containing the air flow inside the operated nasal cavity. Indeed, instantaneous vorticity shown in Figures 7 and 8 points to the existence of small-scale vortical structures and does not contain evidence for large-scale coherent motions.

Thanks to our DNS approach, the analysis based on the instantaneous fields has provided interesting insights into the pathophysiology of radical sinus surgery. The velocity field obviously reflects the flow rate changes and additionally provides spatial detail. As shown in Figures 2 and 3, in an untreated patient, the highest velocity is observed along the middle meatus. In the EMM model, the velocity in the contralateral side decreases, together with the flow rate, and higher velocities are observed in the lateral and cranial portions of the MS. This may decrease the effectiveness of the nose air conditioning effect in terms of humidification and warming. M-EMM reduces these effects, leaving the untreated side entirely unaffected and does not induce any velocity changes inside the MS.

The analysis of the flow unsteadiness, only possible with a DNS simulation, is carried out for the first time in the context of maxillectomy and leads to a most interesting observation. The RMS value of the fluctuations of the velocity magnitude grows by an order of magnitude from the untreated model (where it is nearly negligible, in a steady laminar flow) to the maxillectomy models. The increase of the turbulent kinetic energy $(\mathrm{K})$ volume integral, in both sides, patently higher in the EMM model than in the M-EMM model, further confirms 
the increase of turbulence after surgery. Looking at Figures 5 and 6 , the largest fluctuations are located in the former ethmoid/middle turbinate region, and Figure 8 relates them to small-scale vortical structures present in the flow. In our experience, but also in some case series, ${ }^{2,9}$ this is also the location where most early postoperative crusting occurs. Therefore, our hypothesis is that a highly fluctuating flow field may induce local crusting as a result of the large and fluctuating shear stress. In the M-EMM model, the maximum of the fluctuations is found just behind the IT stump, far from mucosal boundaries. M-EMM could therefore reduce crusting - indeed often a temporary issue in EMM - by diverting large-scale highly oscillating flow structures from the mucosa. It would be extremely interesting to further investigate to what extent high levels of velocity fluctuations and shear stress are connected with crusting also in other sinonasal surgical procedures.

Our analysis last focused on vorticity. As already shown in Figure 7, in the baseline model, the vorticity in the MS is negligible. Vorticity values progressively grow toward the lateral portion of the MS from the M-EMM model to the EMM model. While M-EMM contains disruptions toward the midline, EMM sees intense vorticity fluctuations near the inferior and lateral MS walls. It is interesting to observe that the scarring and partial progressive concentric cavity closure that usually accompany EMM occurs from those very walls, where we might suppose a contribution from shear stress in scar tissues formation. Furthering our speculations, the formation of scar tissue induced by wall shear stress might also induce postsurgical nasal cavity remodeling, which would progressively lead to crusting and scabbing reduction thanks both to a more favorable geometry and the higher tissue resilience. These observations are consistent with other studies on the postoperative MS, which showed in vivo how a higher wall shear stress correlates with residual crusting and discomfort. ${ }^{32}$

The present data, though based on a single-patient analysis, shed light on the fluid dynamics that characterizes radical sinus surgeries. In light of our findings, transnasal endoscopic partial maxillectomy types 1 and 2 have a theoretical CFD basis for their lower overall functional complication rate. ${ }^{33}$ The computational cost prevents us from currently providing a dynamic model integrating different types of turbinate resections. Undoubtedly such model could help understanding nuanced variation in nasal airflow, therefore helping in dosing the turbinate resection as to provide the best surgical field visualization with the slightest fluid dynamics alteration. However, high-fidelity computational models addressing 3 radically different anatomical settings ( 2 of which consisting in commonly employed surgical techniques for EMM) facilitate understanding the overall changes in fluid dynamics in the nasal cavity.

Our work is obviously limited in as much as it considers a single anatomy. Hence, its results cannot be immediately generalized, although much care was devoted to identify a subject whose baseline anatomy is devoid of morphologic alterations and nasal symptoms as to be representative of a normal nose. However, our innovative computational approach uniquely provides access to information free from modeling error and which includes time dependency. Despite such limitations, in view of the rigorous methodology and robust modeling employed, the limited statistical significance of the study is in our opinion more than balanced by the availability of new and quantitatively reliable information.

\section{Acknowledgments}

The Italian Supercomputing Center CINECA has supported this work with computing time provided the Iscra $\mathrm{C}$ project ONOSE-MS. We also gratefully acknowledge the Serpero Foundation (Milan, Italy) for its support.

\section{Declaration of Conflicting Interests}

The author(s) declared no potential conflicts of interest with respect to the research, authorship, and/or publication of this article.

\section{Funding}

The author(s) disclosed receipt of the following financial support for the research, authorship, and/or publication of this article: The Charitable Trust Istituto Farmacologico Filippo Serpero Foundation (Milan, Italy) provided economic support to our group by co-financing the research project.

\section{ORCID iDs}

Alberto M. Saibene (D) https://orcid.org/0000-0003-1457-6871 Antonio Mario Bulfamante (D) https://orcid.org/0000-00031812-6114

\section{References}

1. Kamel RH. Transnasal endoscopic medial maxillectomy in inverted papilloma. Laryngoscope. 1995;105:847-853.

2. Erbek SS, Koycu A, Buyuklu F. Endoscopic modified medial maxillectomy for treatment of inverted papilloma originating from the maxillary sinus. J Craniofac Surg. 2015;26:e244-246.

3. Pagella F, Pusateri A, Giourgos G, et al. Evolution in the treatment of sinonasal inverted papilloma: pedicle-oriented endoscopic surgery. Am J Rhinol Allergy. 2014;28:75-81.

4. Goudakos JK, Blioskas S, Nikolaou A, et al. Endoscopic resection of sinonasal inverted papilloma: systematic review and meta-analysis. Am $J$ Rhinol Allergy. 2018;32:167-174. 
5. Busquets JM, Hwang PH. Endoscopic resection of sinonasal inverted papilloma: a meta-analysis. Otolaryngol Head Neck Surg. 2006;134:476-482.

6. Bertazzoni G, Accorona R, Schreiber A, et al. Postoperative long-term morbidity of extended endoscopic maxillectomy for inverted papilloma. Rhinology. 2017;55:319-325.

7. Pagella F, Pusateri A, Matti E, et al. "TuNa-saving” endoscopic medial maxillectomy: a surgical technique for maxillary inverted papilloma. Eur Arch Otorhinolaryngol. 2017;274:2785-2791.

8. Ghosh A, Pal S, Srivastava A, et al. Modification of endoscopic medial maxillectomy: a novel approach for inverted papilloma of the maxillary sinus. $J$ Laryngol Otol. 2015;129:159-163.

9. Nakayama T, Asaka D, Okushi T, et al. Endoscopic medial maxillectomy with preservation of inferior turbinate and nasolacrimal duct. Am $J$ Rhinol Allergy. 2012;26:405-408.

10. Weber RK, Werner JA, Hildenbrand T. Endonasal endoscopic medial maxillectomy with preservation of the inferior turbinate. Am J Rhinol Allergy. 2010;24:132-135.

11. Chen XB, Lee HP, Chong VFH, et al. Numerical simulation of the effects of inferior turbinate surgery on nasal airway heating capacity. Am J Rhinol Allergy. 2010;24: e118-122.

12. Chen XB, Leong SC, Lee HP, et al. Aerodynamic effects of inferior turbinate surgery on nasal airflow-a computational fluid dynamics model. Rhinology. 2010;48:394-400.

13. Wang F, Yang Y, Wang S, et al. Management of maxillary sinus inverted papilloma via endoscopic partial medial maxillectomy with an inferior turbinate reversing approach. Eur Arch Otorhinolaryngol. 2017;274:4155-4159.

14. Lindemann J, Brambs H-J, Keck T, et al. Numerical simulation of intranasal airflow after radical sinus surgery. Am J Otolaryngol. 2005;26:175-180.

15. Qian Y, Qian H, Wu Y, et al. Numeric simulation of the upper airway structure and airflow dynamic characteristics after unilateral complete maxillary resection. Int $J$ Prosthodont. 2013;26:268-271.

16. Hariri BM, Rhee JS, Garcia GJM. Identifying patients who may benefit from inferior turbinate reduction using computer simulations. Laryngoscope. 2015;125:2635-2641.

17. Pérez-Mota J, Solorio-Ordaz F, Cervantes-de Gortari J. Flow and air conditioning simulations of computer turbinectomized nose models. Med Biol Eng Comput. 2018;56:1899-1910.

18. Weller HG, Tabor G, Jasak H, et al. A tensorial approach to computational continuum mechanics using objectoriented techniques. Comput Phys. 1998;12:620.

19. Covello V, Pipolo C, Saibene A, et al. Numerical simulation of thermal water delivery in the human nasal cavity. Comput Biol Med. 2018;100:62-73.
20. Buijs EFM, Covello V, Pipolo C, et al. Thermal water delivery in the nose: experimental results describing droplet deposition through computational fluid dynamics. Acta Otorhinolaryngol Ital. 2019;39:396-403. In press. doi:10.14639/0392-100X-2250.

21. Fedorov A, Beichel R, Kalpathy-Cramer J, et al. 3D Slicer as an image computing platform for the Quantitative Imaging Network. Magn Reson Imaging. 2012;30:1323-1341.

22. Quadrio M, Pipolo C, Corti S, et al. Effects of CT resolution and radiodensity threshold on the CFD evaluation of nasal airflow. Med Biol Eng Comput. 2016;54:411-419.

23. Quadrio M, Pipolo C, Corti S, et al. Review of computational fluid dynamics in the assessment of nasal air flow and analysis of its limitations. Eur Arch Otorhinolaryngol. 2014;271:2349-2354.

24. Jeong J, Hussain F. On the identification of a vortex. J Fluid Mech. 1995;295:69-94.

25. Keck T, Leiacker R, Heinrich A, et al. Humidity and temperature profile in the nasal cavity. Rhinology. 2000;38:167-171.

26. Lindemann $J$, Kühnemann $S$, Stehmer $V$, et al. Temperature and humidity profile of the anterior nasal airways of patients with nasal septal perforation. Rhinology. 2001;39:202-206.

27. Garcia GJM, Bailie N, Martins DA, et al. Atrophic rhinitis: a CFD study of air conditioning in the nasal cavity. J Appl Physiol. 2007;103:1082-1092.

28. Kastl KG, Rettinger G, Keck T. The impact of nasal surgery on air-conditioning of the nasal airways. Rhinology. 2009;47:237-241.

29. Frank DO, Zanation AM, Dhandha VH, et al. Quantification of airflow into the maxillary sinuses before and after functional endoscopic sinus surgery. Int Forum Allergy Rhinol. 2013;3:834-840.

30. Kahana-Zweig R, Geva-Sagiv M, Weissbrod A, et al. Measuring and characterizing the human nasal cycle. PLoS One. 2016;11:e0162918.

31. Hanna LM, Scherer PW. A theoretical model of localized heat and water vapor transport in the human respiratory tract. J Biomech Eng. 1986;108:19.

32. Choi KJ, Jang DW, Ellison MD, et al. Characterizing airflow profile in the postoperative maxillary sinus by using computational fluid dynamics modeling: a pilot study. Am J Rhinol Allergy. 2016;30:29-36.

33. Turri-Zanoni $\mathrm{M}$, Battaglia $\mathrm{P}$, Karligkiotis $\mathrm{A}$, et al. Transnasal endoscopic partial maxillectomy: operative nuances and proposal for a comprehensive classification system based on 1378 cases. Head Neck. 2017;39:754-766. 